\title{
Management of high-risk non-ST elevation myocardial infarction in the UK: need for alternative models of care to reduce length of stay and admission to angiography times
}

\author{
Authors: Sudheer Koganti ${ }^{A}$ and Roby D Rakhit ${ }^{B}$
}

\begin{abstract}
The roll out of the primary percutaneous coronary intervention pathway as the default treatment for patients with ST elevation myocardial infarction (STEMI) across the NHS has led to a paradigm shift in the model of care resulting in a significant improvement in mortality. In comparison, a similar care plan does not exist for non-ST elevation acute coronary syndrome (NSTE-ACS) despite the fact that patients presenting with high-risk non-STEMI carry a similar if not higher mortality at six months in comparison to STEMI. In this article we focus on the contemporary management of NSTE-ACS in the NHS and also look at some of the dedicated pathways already developed and implemented successfully in expediting treatment and decreasing hospital stay without compromising the safety of patients.
\end{abstract}

KEYWORDS: NSTE-ACS, NSTEMI, dedicated pathways, NHS, hospital stay

\section{Introduction}

Coronary artery disease (CAD) remains the leading cause of death worldwide. ${ }^{1}$ Although the number of people being diagnosed with CAD has plateaued in the western world, it is projected to increase significantly in the developing world by $2020 .^{2}$ Clinical manifestations of CAD include stable angina, acute coronary syndromes (ACS), heart failure and sudden death. The term ACS encompasses ST elevation myocardial infarction (STEMI), non-STEMI (NSTEMI) and unstable angina (UA). The latter two, NSTEMI and UA, are now together described as non-ST elevation ACS (NSTE-ACS). The difference between NSTEMI and UA is lack of evidence of myocardial necrosis in the latter as indicated by absence

Authors: ${ }^{\text {A }}$ clinical research fellow, Department of Cardiology, Royal Free Hospital, London, UK, and UCL Institute of Cardiovascular Science, London, UK; ${ }^{\text {B }}$ consultant interventional cardiologist and honorary senior lecturer, Department of Cardiology, Royal Free Hospital, London, UK, and UCL Institute of Cardiovascular Science, London, UK of cardiac enzyme elevation. The recommended treatment for STEMI is by primary percutaneous coronary intervention (PPCI), which has been proved to be more effective than thrombolytic therapy. ${ }^{3}$ The roll out of PPCI as the default treatment for patients with STEMI across the NHS has led to a paradigm shift in the model of care resulting in a significant improvement in mortality. ${ }^{4}$ In comparison, a similar care plan does not exist for NSTE-ACS despite the fact that patients presenting with high-risk NSTEMI carry a similar if not higher mortality at six months ${ }^{5}$ in comparison to STEMI. The lack of a unifying pathway for management of NSTE-ACS patients similar to PPCI is due to i) heterogeneity of the case mix; ii) presence of comorbidities; and iii) lack of adherence to clearcut guidelines. Consequently, patients with NSTE-ACS can be admitted to hospitals without cardiac catheter laboratory facilities, resulting in delayed treatment and increased length of hospital stay. Even patients admitted to hospitals which offer coronary intervention face similar delays as they await cardiology referral and review. In this article we focus on the contemporary management of NSTE-ACS in the NHS, and look at some of the dedicated pathways already developed and implemented successfully in expediting treatment and decreasing hospital stay without compromising the safety of patients.

\section{Epidemiology}

The Myocardial Infarction National Audit Project (MINAP) set up in 1999 audits ACS patients and the treatment they receive in England, Wales and Belfast. According to the MINAP report for the year 2012/2013 there were 80,974 admissions with ACS across the NHS. This number includes 32,665 (40\%) STEMI and 48,309 (60\%) NSTEMI cases. However, not all NSTEMI cases are recorded in the MINAP database due to underreporting. The commonly held assumption is that for every case of STEMI there are three admissions related to NSTEMI. If this 1:3 ratio is applied to all ACS cases and recalculated, the projected number of NSTEMI cases should be well over 100,000 per annum. Furthermore, patients presenting with NSTE-ACS tend to be older, frequently have multivessel disease and have complex comorbid medical history. 


\section{Evidence behind current medical management of patients with NSTE-ACS}

In addition to emergency assessment, haemodynamic stabilisation and symptom relief of patients presenting with chest pain secondary to NSTE-ACS, it is also paramount that evidence-based medical therapy is commenced without delay.

Current guideline-based medical therapy includes dual antiplatelet therapy with aspirin, thienopyridines (eg clopidogrel, prasugrel) or triazolopyrimidines (ticagrelor), low molecular weight heparin or factor Xa inhibitors such as fondaparinux and or glycoprotein IIb/IIIa inhibitors (abciximab, tirofiban and eptifibatide). Individual patient bleed risk needs to be taken into account prior to commencing the above-mentioned drugs. Glycoprotein IIb/IIIa inhibitors are not prescribed routinely and are reserved for high-risk patients requiring coronary intervention.

Risk stratification of patients presenting with NSTE-ACS is important as it predicts short- and medium-term mortality and can be utilised as a guide to the cardiologist in planning the timing of coronary angiography with or without intervention. The Global Registry of Acute Coronary Events (GRACE) score accurately predicts the risk of recurrent myocardial infarction and death at 30 days and six months. ${ }^{6}$ Integrated care pathway documents for NSTE-ACS incorporate GRACE scoring tools and are easy to calculate as they are based on simple clinical and biochemical parameters. Once medical therapy is established and risk stratification is carried out, the decision regarding revascularisation is taken. NSTE-ACS patients with ongoing chest pain and haemodynamic instability should be considered for immediate coronary angiography, as recommended by guidelines from the European Society of Cardiology ${ }^{7}$ (Box 1). However, the optimal timing of angiography (and follow-on

Box 1. ESC recommendations for initial evaluation and angiography. ${ }^{7}$

> An invasive strategy (within $72 \mathrm{~h}$ ) after first presentation is indicated in patients with at least one high-risk criterion (Box 2) and or recurrent symptoms.

$>$ Urgent coronary angiography $(<2 \mathrm{~h})$ is recommended in patients at very high ischaemic risk (refractory angina, associated heart failure, life-threatening ventricular arrhythmias or haemodynamic instability).

$>$ An early invasive strategy $(<24 \mathrm{~h})$ is recommended with a GRACE score $>140$ or with at least one primary high-risk criterion.

> Non-invasive documentation of inducible ischemia is recommended in low-risk patients without recurring symptoms before deciding for invasive evaluation.

> Likewise routine invasive evaluation of low-risk groups is not recommended. However, if there is a secondary high-risk criterion (Box 2) present in low-risk subset with GRACE <140, invasive evaluation can be delayed but is recommended to be carried out during the same hospital stay or preferably within $72 \mathrm{~h}$ of admission.

ESC $=$ European Society of Cardiology; GRACE = Global Registry of Acute Coronary Events.
Box 2. High-risk criteria set out by ESC in the

management and for evaluation of invasive angiography in patients presenting with NSTEMI. ${ }^{7}$

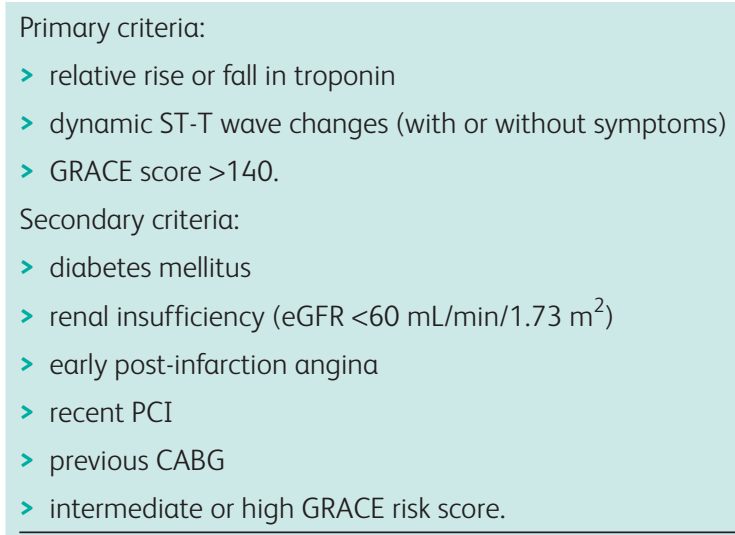

NB: This is different to the GRACE score (for GRACE score visit gracescore. co.uk). $C A B G$ = coronary artery bypass surgery; eGFR = estimated glomerular filtration rate; ESC = European Society of Cardiology; GRACE = Global Registry of Acute Coronary Events; $\mathrm{PCI}=$ percutaneous coronary intervention

PCI) for NSTE-ACS patients without ongoing chest pain or haemodynamic instability remains unclear. However, updated guidelines from the National Institute for Health and Care Excellence (NICE) recommend invasive treatment within 72 hours of first hospital admission ${ }^{8}$ for those at intermediate risk and above; this represents the majority of patients presenting with NSTE-ACS. The evidence for a routine invasive strategy compared to a conservative approach is now unequivocal. Meta-analysis of several randomised studies including FRagmin and Fast Revascularisation during InStability in Coronary artery disease (FRISC-2), Invasive versus Conservative Treatment in Unstable Coronary Syndromes (ICTUS), and Randomized Intervention Trial of unstable Angina-3 (RITA3) studies showed a reduction in mortality and non-fatal MI at five-year follow up in the routine invasive strategy group, with the most pronounced difference in high-risk patients. ${ }^{9}$ Furthermore, meta-analysis of Angioplasty to Blunt the Rise of Troponin in Acute Coronary Syndromes Randomised for an Early or Delayed Intervention (ABOARD), Timing of Intervention in Patients with Acute Coronary Syndromes (TIMACS), Intracoronary Stenting With Antithrombotic Regimen Cooling Off (ISAR-COOL) and Early or Late Intervention in unStable Angina (ELISA) trials have shown early invasive strategy to be safe, effective and reduce the length of hospital stay. ${ }^{10}$ Finally the decision to revascularise either with PCI or coronary artery bypass grafting (CABG) is based on the extent of disease seen on angiography and the presence of other comorbidities.

\section{Current model of care for NSTE-ACS in the NHS}

The delivery of care for patients with NSTE-ACS differs significantly to those with STEMI (Fig 1). PPCI pathways designed to bring patients with STEMI direct to heart attack centres result in better outcomes. However, despite similar six-month mortality, such treatment pathways do not exist 

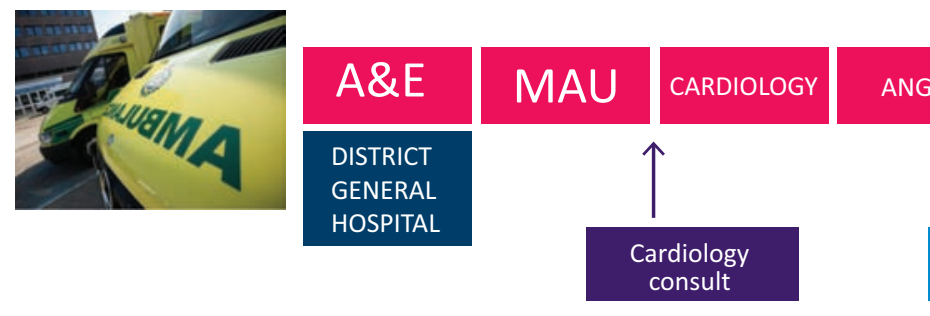

Day 1

Day 2

Day 3

$>$ Day 4

Day 5

Day 6

Fig 1. Current pathway for NSTE-ACS in the UK for DGH with angiogram only facilities. DGH = district general hospital; IHT = interhospital transfer; $\mathrm{MAU}=$ medical assessment unit; NSTE-ACS = non-ST elevation acute coronary syndrome; $\mathrm{PCI}=$ percutaneous coronary intervention.

for patients presenting with high-risk NSTE-ACS. Most patients with NSTE-ACS in the UK are admitted to a district general hospital (DGH) without cardiac catheter laboratory facilities. ${ }^{11}$ Similarly, a proportion of patients with NSTEACS are admitted under the general medical take at a tertiary centre pending assessment by the cardiology team. Both pathways lead to delay in patients accessing optimised medical management and angiography, while increasing length of hospital stay. According to the 2013/2014 MINAP report, 67\% of patients admitted with NSTE-ACS received angiography within 96 hours of admission and only 55\% received angiography within 72 hours of first hospital admission; the target stipulated by the current NICE guideline. ${ }^{12}$ Looking further into numbers, in the year 2013/14, median length of stay for patients with NSTEMI in England and Wales was 5 days (interquartile range (IQR) 3-9 and 3-10 respectively). This does not include interhospital transfers, thus patients admitted to a DGH without a cardiac catheter laboratory may end up staying even longer in the hospital. ${ }^{12}$

\section{Role of facilitated pathways in treating patients with NSTE-ACS}

Removing the inherent delay involved with the general medical pathway and facilitating early triage, assessment by cardiologists and access to PCI are key to streamlining the pathway for high-risk NSTEACS patients. We illustrate this by describing three novel pathways (including our own), which have been developed to fast track high-risk patients to a heart attack centre for early angiography and PCI (Fig 2). The Heart
Attack Centre-Extension (HAC-X) pathway implemented at the London Chest Hospital in 2010 involves triage of patients in accident and emergency (A\&E) presenting with NSTE-ACS and transfer via a dedicated ambulance directly to the Heart Attack Centre. In 2010, a pan-London high-risk ACS pathway was proposed and implemented in 2011 across London as part of a cardiovascular review ${ }^{13}$ highlighting the need to identify high-risk NSTE-ACS using key clinical features and stating the need for guaranteed access within 24 hours to a 24/7 PCI centre. In 2011 we developed a direct access pathway (DAP) at the Royal Free Hospital, enabling London Ambulance Service (LAS) crew to bring patients with high-risk NSTE-ACS (chest pain within 12 hours and high-risk electrocardiogram (ECG) features) from home directly to the heart attack centre for GRACE risk assessment and early angiography. The data show an overwhelming reduction in time to angiography and duration of in-hospital stay.

The median length of in-hospital stay for patients admitted by HAC-X pathway was 3 days (IQR 3-6) and the median time from $A \& E$ admission to coronary angiography was 1.0 (IQR 0.7-2.0) day. ${ }^{14}$ A local audit of pan-London pathway versus DAP patients in our institution ${ }^{15}$ has similarly shown a median length of stay of 3 days (IQR 3-5) and median time to angiography of 16.6 hours (IQR 6-50) for the pan-London pathway and 2.8 hours (IQR 1.5-9) for DAP. In comparison to national data this represents a reduction of 2 in patient bed days per patient. A significant reduction in times was achieved by removing the traditional bottlenecks that result in delay while admitting and treating patients with NSTE-ACS via A\&E and the general medical pathway.

\section{HAC-X pathway}

\section{I}

NSTE-ACS diagnosis confirmed at DGH A\&E department

Transfer to Heart Attack Centre (London Chest Hospital) within $1 \mathrm{~h}$

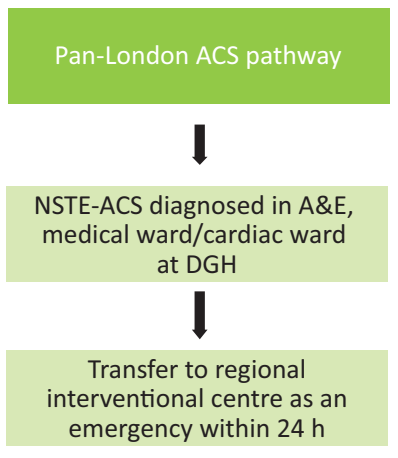

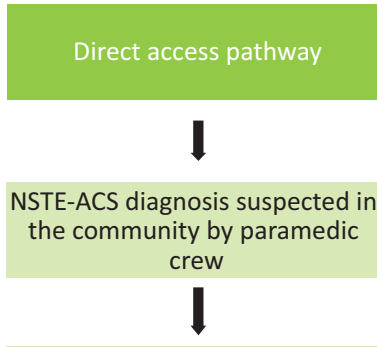

Transfer directly to Heart Attack Centre (Royal Free Hospital)
Fig 2. Flowchart showing different pathways. $A \& E=$ accident and emergency; $\mathrm{DGH}=$ district general hospital; $\mathrm{HAC}-\mathrm{X}=$ heart attack extension pathway; NSTEACS = non-ST elevation acute coronary syndrome. 


\section{Advantages of a direct access pathway}

The DAP was set up in 2011 and implemented with the aim of delivering high-risk NSTE-ACS patients directly to cardiologists for risk assessment and evidence-based treatment. Paramedic LAS staff were trained to undertake the index assessment for the activation of this pathway (this included recognition of high-risk ECG features such as ST depression or T-wave inversion). Troponin elevation was not included in the activation criteria to reduce delay and allow activation of the pathway in the community. Upon activation, the patient is transferred directly to the Heart Attack Centre similar to PPCI for STEMI. A consultant cardiologist or a cardiology registrar receives these patients and decision to perform urgent angiography is made. Patients deemed to be high risk, but without ongoing chest pain were admitted directly to a monitored cardiac ward, commenced on evidencebased therapy and angiography was performed within 24 hours. All other patients underwent immediate angiography. Implementing DAP at the Royal Free Hospital has resulted in a significant reduction in time of admission to angiography and in-hospital stay when compared to existing model of care (unpublished data). Furthermore this pathway was safe, feasible and the LAS appear to be good discriminators.

\section{Limitations of fast-track pathways}

A key limitation of implementing these novel pathways for the management of NSTE-ACS is the demand for resources at the heart attack centres which are already busy with a heavy STEMI caseload. This demand includes staffing, beds and competition for elective cases. Patients with NSTE-ACS who were found to have surgical disease end up staying within tertiary centres longer prior to CABG; this increases further pressure on bed availability. However, increasing demand for beds at an interventional centre may be offset by decreasing the pressure on beds at the local DGH while reducing a double acute tariff currently being paid for by commissioners. However, there may be a need for heart attack centres to enlarge to being ACS centres which are adequately resourced with an improved bed base. Lessons can be learned from the hyperacute stroke model for stroke thrombolysis in the UK. ${ }^{16}$

\section{Conclusion}

Management of patients presenting with NSTE-ACS has improved significantly over the last decade. An increasing number of patients are referred for angiography, seen by cardiologists and put on secondary prevention medication. However, only one-half of patients in the UK at intermediate or high risk currently receive timely angiography and PCI according to current guidelines, due to intrinsic delays in the model of service delivery. Patients presenting with high-risk features have a high mortality similar to STEMI and there is a need to facilitate access to angiography and PCI for this patient group. Novel pathways which provide a systematic approach to early diagnosis, risk stratification and consideration of 'direct transfer' of patients with NSTE-ACS to an interventional cardiac ('heart attack centre') have the potential to achieve better results and outcomes. Reducing overall length of stay for this group of patients and extrapolating this to the UK will have a significant impact on relieving an already stretched nonelective urgent care pathway.

\section{References}

1 Murray CJ, Lopez AD. Alternative projections of mortality and disability by cause 1990-2020: Global Burden of Disease Study. Lancet 1997;349:1498-504.

2 World Health Organization. Global status report on noncommunicable diseases. Geneva: WHO, 2011.

3 Keeley EC, Boura JA, Grines CL. Primary angioplasty versus intravenous thrombolytic therapy for acute myocardial infarction: a quantitative review of 23 randomised trials. Lancet 2003;361:13-20.

4 Myocardial Ischaemia National Audit Project. How the NHS cares for patients with heart attack. London: MINAP, 2010.

5 Allen LA, O’Donnell CJ, Camargo CA Jr, Giugliano RP, Lloyd-Jones DM. Comparison of long-term mortality across the spectrum of acute coronary syndromes. Am Heart J 2006;151:1065-71.

6 Fox KA, Dabbous OH, Goldberg RJ et al. Prediction of risk of death and myocardial infarction in the six months after presentation with acute coronary syndrome: prospective multinational observational study (GRACE). BMJ 2006;333:1091.

7 Hamm CW, Bassand JP, Agewall S et al. ESC guidelines for the management of acute coronary syndromes in patients presenting without persistent ST-segment elevation: the task force for the management of acute coronary syndromes (ACS) in patients presenting without persistent ST-segment elevation of the European Society of Cardiology (ESC). Eur Heart J 2011;32:2999-3054.

8 National Institute for Health and Care Excellence. Unstable angina and NSTEMI: The early management of unstable angina and nonST-segment-elevation myocardial infarction. London: NICE, 2010. Available online at www.nice.org.uk/guidance/cg94 [Accessed 21 September 2015].

9 Fox KA, Clayton TC, Damman P et al. Long-term outcome of a routine versus selective invasive strategy in patients with non-STsegment elevation acute coronary syndrome a meta-analysis of individual patient data. J Am Coll Cardiol 2010;55:2435-45.

10 Katritsis DG, Siontis GC, Kastrati A et al. Optimal timing of coronary angiography and potential intervention in non-ST-elevation acute coronary syndromes. Eur Heart J 2011;32:32-40.

11 Bellenger NG, Eichhofer J, Crone D, Curzen N. Hospital stay in patients with non-ST-elevation acute coronary syndromes. Lancet 2004;363:1399-400.

12 Myocardial Ischaemia National Audit Project. How the NHS cares for patients with heart attack. London: MINAP, 2014. Available online at www.ucl.ac.uk/nicor/audits/minap/documents/annual_ reports/minap-public-report-2014 [Accessed 21 September 2015].

13 London Health Programmes. Cardiovascular project proposed model of care, August 2010. Available online at www.londonhp.nhs.uk/ wp-content/uploads/2011/03/Cardiovascular-model-of-care.pdf [Accessed 21 September 2015].

14 Gallagher SM, Lovell MJ, Jones DA et al. Does a 'direct' transfer protocol reduce time to coronary angiography for patients with non-ST-elevation acute coronary syndromes? A prospective observational study. BMJ Open 2014;4:e005525.

15 Koganti S, Seraphim A, Rakhit R. Feasibility of a fast track pathway for high risk ACS: effects on angiography times, length of stay and mortality. Heart 2015;101:A13.

16 Liu SD, Rudd A, Davie C. Hyper acute stroke unit services. Clin Med 2011;11:213-4.

Address for correspondence: Dr RD Rakhit, Royal Free Hospital and UCL Institute of Cardiovascular Science, Pond Street, London NW3 2QG, UK.

Email: roby.rakhit@nhs.net 